\title{
Cigarette smoke extract-induced proliferation of normal human urothelial cells via the MAPK/AP-1 pathway
}

\author{
HAO GENG $^{1 *}$, LI ZHAO $^{1 *}$, ZHAOFENG LIANG $^{2}$, ZHIQIANG ZHANG $^{1}$, DONGDONG XIE $^{1}$, \\ LIANGKUAN BI $^{1}$, YI WANG ${ }^{1}$, TAO ZHANG ${ }^{1}$, LEI CHENG ${ }^{1}$, DEXIN YU $^{1}$ and CAIYUN ZHONG ${ }^{2}$ \\ ${ }^{1}$ Department of Urology, The Second Hospital of Anhui Medical University, Hefei, Anhui 230032; \\ ${ }^{2}$ Department of Nutrition and Food Safety, School of Public Health, \\ Nanjing Medical University, Nanjing, Jiangsu 211166, P.R. China
}

Received February 26, 2015; Accepted June 16, 2016

DOI: $10.3892 / 01.2016 .5407$

\begin{abstract}
Bladder cancer (BC) is universally acknowledged as a significant public health issue, worldwide. Numerous studies have demonstrated that cigarette smoke is the primary risk factor for $\mathrm{BC}$. However, the mechanism of cigarette smoke-induced BC has not been fully elucidated. Sustained epithelial cell hyperplasia has been identified as a preneoplastic lesion during the formation of $\mathrm{BC}$. The aim of the present study was to investigate whether exposure to cigarette smoke extract (CSE) induced proliferation in normal human urothelial SV-HUC-1 cells. Furthermore, the role of the mitogen-activated protein kinase (MAPK)/activator protein-1 (AP-1) pathway in the CSE-induced proliferation of SV-HUC-1 cells was also investigated. The present study revealed that the expression of phosphorylated-extracellular signal regulated protein kinase (ERK)1/2, Jun N-terminal kinase (JNK) and p38 was significantly increased following exposure to CSE in SV-HUC-1 cells. Furthermore, CSE increased the expression of the proliferation markers, cyclin D1 and proliferating cell nuclear antigen. By contrast, CSE attenuated the expression of p21. In addition, the inhibitors of ERK1/2 and JNK reversed the aforementioned effects of CSE. However, p38 inhibition did not reverse CSE-induced proliferation. In conclusion, the results of the present study demonstrated that exposure to CSE induced proliferation in normal human urothelial cells.
\end{abstract}

Correspondence to: Mr. Dexin Yu, Department of Urology, The Second Hospital of Anhui Medical University, 678 Furong Road, Hefei, Anhui 230032, P.R. China

E-mail: yudx_urology@126.com

Mr. Caiyun Zhong, Department of Nutrition and Food Safety, School of Public Health, Nanjing Medical University, 818 Tianyuan East Road, Nanjing, Jiangsu 211166, P.R. China

E-mail: cyzhong@njmu.edu.cn

*Contributed equally

Key words: bladder cancer, cigarette smoke extract, proliferation, mitogen-activated protein kinase/activator protein-1 pathway
Furthermore, the results also indicated that the ERK1/2 and JNK pathways are important for the regulation of proliferation via the AP-1 proteins.

\section{Introduction}

Bladder cancer (BC) is the fifth most common type of cancer in Western countries, accounting for $>130,000$ mortalities annually, worldwide (1). At any point in time, 2.7 million people are diagnosed with or have a history of BC (1).

Cigarette smoke is the major risk factor for $\mathrm{BC}$ (2). Recently, a meta-analysis of 43 published case-control and cohort studies reported that, at present, cigarette smokers exhibit a $\sim 3$-fold higher risk of developing $\mathrm{BC}$ compared with non-smokers (3). The risk of $\mathrm{BC}$ has been found to significantly correlate with the intensity and duration of cigarette smoke exposure, whereas quitting smoking reduces this risk (4).

Exposure to CSE or its constituents is known to trigger a cascade of events in the multistage process of carcinogenesis (5). A previous study revealed that the mitogen-activated protein kinase (MAPK)/activator protein-1 (AP-1) pathway is associated with the effects induced by CSE (6). MAPKs are involved in the phosphorylation and activation of the Jun and Fos proteins. Three well-characterized subfamilies of MAPK have been identified, which include the extracellular signal-regulated kinase (ERK)1/2, Jun N-terminal kinase (JNK)/stress-activated protein kinase and p38 subfamilies (7). The MAPK signaling pathway, which is upregulated in cancer cells, promotes proliferation, differentiation and cell survival, and mediates oncogenesis (8).

Malignant tumors exhibit aberrant cell growth, which results from uncontrolled cell division and proliferation. Urothelial hyperplasia has been identified as a precancerous lesion in BC (9). Exposure to nicotine induces the expression of cyclin D1 and proliferating cell nuclear antigen (PCNA), which are two AP-1 target genes known to be involved in promotion of cell cycle progression (10). Cyclin D1 monitors the cell cycle by forming complexes with cyclin-dependent kinase 4 and 6 in the cytoplasm. These complexes then enter the nucleus and inactivate the cell-cycle suppressive retinoblastoma protein, thereby promoting progression from G1 
to $\mathrm{S}$ phase (10). By contrast, the tumor suppressor, $\mathrm{p} 21$, is a key negative regulator of the cell cycle and cell proliferation, and is often downregulated in cancer. Notably, p21 is rarely mutated or deleted (11). However, few studies have investigated the effect of CSE on the cell cycle in normal human urothelial cells.

Thus, the aim of the present study was to investigate whether exposure to CSE induced proliferation in SV-HUC-1 cells. Furthermore, the role of the MAPK/AP-1 pathway in CSE-induced proliferation of SV-HUC-1 cells was also analyzed.

\section{Materials and methods}

Reagents. The SV-40 immortalized human urothelial SV-HUC-1 cell line was purchased from the Chinese Academy of Sciences Cell Bank (Shanghai, China). Gibco F12K medium was purchased from Thermo Fisher Scientific, Inc. (Waltham, MA, USA). Fetal bovine serum (FBS) was obtained from PAA Laboratories GmbH (Pasching, Austria). 3-(4,5)-dime thylthiahiazo(-z-y1)-3,5-di-phenytetrazoliumromide (MTT) was purchased from Sigma-Aldrich (Merck Millipore, Darmstadt, Germany). Monoclonal rabbit phosphorylated (p-) JNK (cat. no. AF3318; 1:1,000), p38 (cat. no. 9212; 1:1,000), ERK1/2 (cat. no. RS-2637S; 1:1,000), p-c-Jun (cat. no. AF-3095; 1:1,000), p-c-Fos (cat. no. 5348; 1:1,000), Jun B (cat. no. 104861-AP; 1:1,000), p21 (cat. no. 10355-1-AP; 1:1,000), cyclin D1 (cat. no. 2978; 1:1,000) and PCNA (cat. no. 10205-2-AP; 1:1,000) primary antibodies were all purchased from Cell Signaling Technology, Inc. (Danvers, MA, USA). Monoclonal rabbit glyceraldehyde 3-phosphate dehydrogenase (GAPDH; cat. no. 60004-1-Ig; 1:1,000) was purchased from Proteintech (Rosemont, IL, USA). Monoclonal rabbit Jun D antibody (cat. no. sc-2048; 1:1,000) was purchased from Santa Cruz Biotechnology, Inc. (Santa Cruz, CA, USA). Mouse anti-rabbit IgG secondary antibodies (cat. no. bs-0295M; 1:10,0000) were purchased from Saihongrui Biotechnology Co., Ltd., (Nanjing, China). Primers were synthesized according to published sequences by Invitrogen (Thermo Fisher Scientific, Inc.). Inhibitors of the MAPK pathway (SB203580 for p38, SP600125 for JNK and U0126 for ERK) were purchased from Beyotime Institute of Biotechnology (Shanghai, China).

Cell culture and treatment. SV-HUC-1 cells were cultured in F12K medium (Sigma-Aldrich; Merck Millipore) containing antibiotics (100 units $/ \mathrm{ml}$ penicillin and $100 \mu \mathrm{g} / \mathrm{ml}$ streptomycin) in an atmosphere of $5 \% \mathrm{CO}_{2}$ at $37^{\circ} \mathrm{C}$. Cells were seeded in $10 \mathrm{~cm}^{2}$ flasks at a density of $1 \times 10^{5}$ cells per well. The medium was changed every other day until cells reached $80-90 \%$ confluence, then treated with $0,0.10,0.25$ or $0.50 \%$ cigarette smoke extract (CSE) or U0126 (5 $\mu \mathrm{M})$, SB203580 $(5 \mu \mathrm{M})$ or SP600125 (2 $\mu \mathrm{M})$.

Preparation of CSE. CSE was freshly prepared for each experiment by combusting one commercial cigarette, according to a previously reported method (12). Commercial cigarettes (Hongtashan filter-tipped cigarettes; YuXi Cigarette Factory, Yunnan, China), each containing $12 \mathrm{mg}$ tar and $1.1 \mathrm{mg}$ nicotine, were combusted. Using a vacuum, mainstream (first-hand) smoke was drawn through $10 \mathrm{ml}$ pre-warmed $\left(37^{\circ} \mathrm{C}\right) \mathrm{FBS}$-free $\mathrm{F} 12 \mathrm{~K}$ medium supplemented with penicillin and streptomycin at a rate of $5 \mathrm{~min} /$ cigarette (13). The obtained CSE stock solution was considered to contain a concentration of $100 \%$. The CSE stock solution was then filtered through a $0.22-\mu \mathrm{m}$-pore size filter and diluted to the desired concentrations using treatment medium. The resulting CSE solutions were applied to epithelial cell cultures within $30 \mathrm{~min}$ of preparation. A control solution was prepared using the same protocol, using unlit cigarettes.

Cell proliferation assay. SV-HUC-1 cells $\left(2 \times 10^{3}\right.$ cells/well) were seeded in 96-well plates in $200 \mu 1 \mathrm{~F} 12 \mathrm{~K}$ medium. Cells were exposed to $0,0.10,0.25$ or $0.50 \%$ CSE for 7 days and cell viability was then determined by MTT assay. The media were changed every day. Subsequent to 7 days of culture, MTT stock solution $(5 \mathrm{mg} / \mathrm{ml})$ was added to each well to solubilize formazan crystals, and plates were incubated for an additional $4 \mathrm{~h}$ at $37^{\circ} \mathrm{C}$. Next, MTT solution was removed and the crystals were solubilized in dimethyl sulfoxide. Absorbance was measured at $490 \mathrm{~nm}$ using a microplate reader (Titertek Instruments Inc., Huntsville, AL, USA). All experiments were performed in triplicate.

Cell cycle analysis. The cell cycle distribution of SV-HUC-1 cells was determined by flow cytometry. SV-HUC-1 cells $\left(5 \times 10^{5}\right.$ cells/well) were incubated in $\mathrm{F} 12 \mathrm{~K}$ medium supplemented with $5 \% \mathrm{FBS}$ at $37^{\circ} \mathrm{C}$ overnight. Cells were then cultured in F12K medium without FBS for 6 h. Next, cells were treated with $0,0.1,0.25$ and $0.5 \%$ CSE for 7 days. Cells were trypsinized, washed twice with cold phosphate-buffered saline (PBS) and fixed overnight with $70 \%$ ethanol at $4^{\circ} \mathrm{C}$ followed by resuspension in $500 \mu \mathrm{l}$ PBS. Following the addition of $10 \mu \mathrm{l}$ RNAse $(10 \mathrm{mg} / \mathrm{ml})$, cells were incubated for $30 \mathrm{~min}$ in the dark at $37^{\circ} \mathrm{C}$ then stained with $10 \mu \mathrm{l}$ propidium iodide $(1 \mathrm{mg} / \mathrm{ml})$. Cell cycle analysis was then performed by flow cytometry (FACSCalibur instrument; BD Biosciences, San Jose, CA, USA). The percentage of cells in each cell cycle phase (G0/G1, S or G2/M) was calculated using MultiCycle 3.0 software (BD Biosciences). All experiments were performed in triplicate.

Western blot analysis. SV-HUC-1 cells were harvested at 70-80\% confluence, washed with ice-cold PBS and lysed in RIPA buffer (Thermo Fisher Scientific, Inc.). The concentration of precipitated proteins in the cell lysates was measured using the BCA Protein Assay kit (Pierce Biotechnology, Inc., Rockford, IL, USA). Next, proteins were diluted to equal concentrations, boiled for $5 \mathrm{~min}$, separated by $7.5-10.0 \%$ sodium dodecyl sulfate-polyacrylamide gel electrophoresis and transferred to polyvinylidene difluoride membranes (Millipore, Billerica, MA, USA). After blocking with 5\% milk, the membranes were incubated with the p-JNK, p38, ERK1/2, p-c-Jun, p-c-Fos, Jun B, Jun D, p21, cyclin D1 and PCNA primary antibodies at $4{ }^{\circ} \mathrm{C}$ overnight. The membranes were washed with PBS 3 times for 5 min each time, prior to incubation with secondary antibodies for $1 \mathrm{~h}$ at room temperature. The blots were subsequently developed using an enhanced chemiluminescence detection kit (Amersham Biosciences, Uppsala, Sweden) and exposed to film (Kodak, Rochester, NY, USA). GAPDH acted as the loading control. 
A

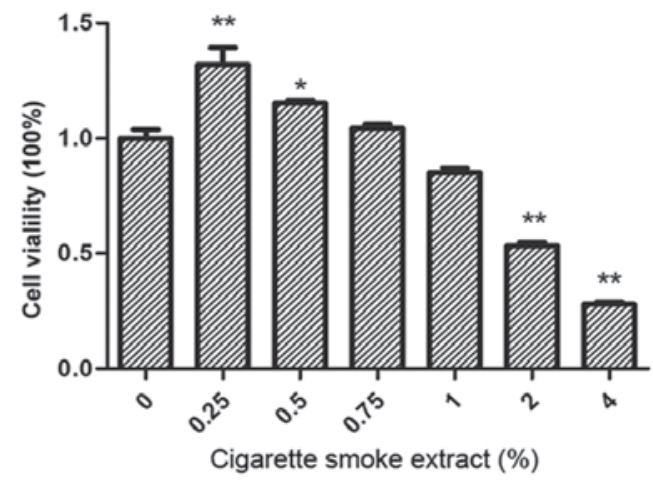

B

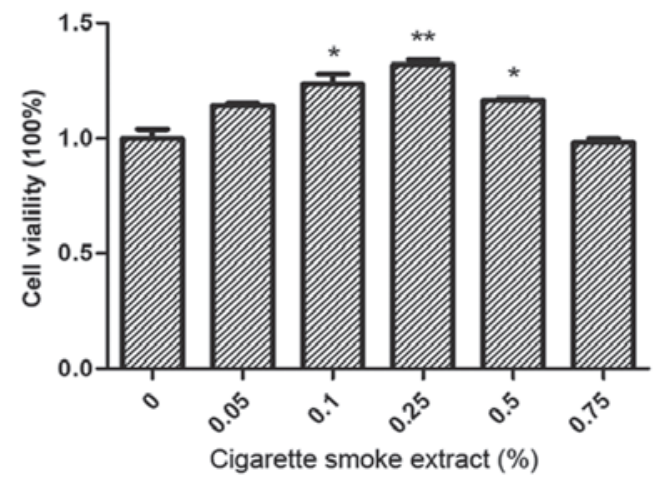

Figure 1. Change in SV-HUC-1 cell viability following treatment with 0, 0.05, 0.10, 0.25, 0.50, 0.75, 1, 2 and 4\% CSE for 7 days. (A) Treatment with 0.25 and $0.50 \%$ CSE significantly increased the proliferation of SV-HUC-1 cells, whereas treatment with 2 and $4 \%$ CSE resulted in a significant decrease in cell viability. (B) Treatment with $0.10 \%$ CSE also promoted proliferation. Data are representative of three independent experiments and are expressed as the mean \pm standard deviation. ${ }^{*} \mathrm{P}<0.05$ and ${ }^{* *} \mathrm{P}<0.01$ vs. control group. CSE, cigarette smoke extract.

Quantitative reverse transcription-polymerase chain reaction ( $q R T-P C R)$. Total cellular RNA was isolated using Trizol reagent (Invitrogen; Thermo Fisher Scientific, Inc.) according to the manufacturer's instructions. Total RNA was reverse-transcribed using the Easy RT-PCR kit (Takara Bio, Inc., Otsu, Japan). qRT-PCR was performed using the Power SYBR Green Master Mix (Applied Biosystems; Thermo Fisher Scientific, Inc.) and an ABI 7300 Real-time PCR Detection System (Applied Biosystems; Thermo Fisher Scientific, Inc.). A $20 \mu \mathrm{l}$ sample system was used, including $1 \mu \mathrm{l}$ cDNA, $0.6 \mu 1$ forward primer, $0.6 \mu 1$ reverse primer, $7.8 \mu \mathrm{l}$ diethylpyrocarbonate water and $10 \mu \mathrm{l}$ Rox-mix. PCR was performed under the following conditions: Denaturation at $95^{\circ} \mathrm{C}$ for $15 \mathrm{sec}$, followed by 40 cycles of annealing at $65^{\circ} \mathrm{C}$ for $30 \mathrm{sec}$ and extension at $72^{\circ} \mathrm{C}$ for $15 \mathrm{sec}$. The sequences of the primers (Invitrogen; Thermo Fisher Scientific, Inc.) used for RT-PCR were as follows: Forward, 5'-CGTGGCCTC TAAGATGAAGG-3' and reverse, 5'-TGCGGATGATCT GTTTGTTC-3' for cyclin D1; forward, 5'-GACACCACT GGAGGGTGACT-3' and reverse, 5'-CAGGTCCACATG GTCTTCCT-3' for p21; and forward, 5-'GCTGCCCAACGC ACCGAATA-3' and reverse, 5'-GAGTCAACGGATTTG GTCGT-3' for GAPDH. Each sample was run in triplicate. Fold changes in the expression of each gene were calculated using the comparative threshold cycle $(\mathrm{Ct})$ method using the formula $2^{-\Delta \Delta \mathrm{Ct}}(14)$.

Statistical analysis. All statistical analysis was performed using SPSS 17.0 (SPSS, Inc., Chicago, IL, USA). Data are expressed as the mean \pm standard deviation. One-way analysis of variance or the Kruskal-Wallis test were used to analyze differences among groups. $\mathrm{P}<0.05$ was considered to indicate a statistically significant difference.

\section{Results}

CSE induces proliferation in SV-HUC-1 cells. To study the cell viability of SV-HUC-1 cells following treatment with CSE, cells were incubated with CSE $(0,0.25,0.50$, $0.75,1,2$ and 4\%) for 7 days and examined by MTT assay. A significant increase in the cell viability of SV-HUC-1 cells following treatment with $0.25(\mathrm{P}<0.001)$ and $0.50 \%$ $(\mathrm{P}=0.042) \mathrm{CSE}$ was identified, while cell viability decreased to $<80 \%$ following treatment with CSE concentrations of $\geq 2 \%$ $(\mathrm{P}<0.001)$, which were demonstrated to be toxic to SV-HUC-1 cells (Fig. 1A). To further investigate the effect of CSE treatment on the proliferation of SV-HUC-1 cells, lower concentrations of CSE (0.25 and $0.50 \%)$ were selected to study cell viability in further experiments. Although a CSE concentration of $0.05 \%$ increased SV-HUC-1 cell proliferation, concentrations of 0.10 , 0.25 and $0.50 \% \mathrm{CSE}$ were selected as the optimal concentrations for future investigation, as they resulted in the most significant differences in proliferation (Fig. 1B).

CSE promotes SV-HUC-1 cell transformation from G1 to $S$ phase. To examine whether the proliferative effects of CSE in SV-HUC-1 cells were mediated via cell cycle modulation, the cell cycle distribution in SV-HUC-1 cells was investigated by flow cytometry. Low concentrations of CSE $(0.10,0.25$ and $0.50 \%$ ) promoted SV-HUC-1 cell transformation from G1 to S phase (Fig. 2A and B). Notably, a total of 39.83\% of SV-HUC-1 cells treated with $0.25 \%$ CSE entered $S$ phase, which was significantly increased compared with the control group (29.82\%; $\mathrm{P}=0.032$ ). Next, the expression of proteins associated with the cell cycle were determined; the results revealed that the expression of cyclin D1 and PCNA were markedly increased following $0.10,0.25$ and $0.50 \%$ CSE treatment. Notably, the expression of p21 was attenuated by CSE treatment and the expression of p21 almost disappeared following treatment with $0.5 \%$ CSE (Fig. 2C-D).

MAPK/AP-1 pathway is involved in the proliferative effects induced by CSE in SV-HUC-1 cells. The MAPK/AP-1 pathway is hypothesized to be involved in the effects induced by CSE in SV-HUC-1 cells. Therefore, SV-HUC-1 cells were treated with $0,0.10,0.25$ or $0.50 \%$ CSE for 7 days followed by western blot analysis to investigate whether CSE activates the MAPK/AP-1 pathway. The results revealed that CSE activated p-ERK1/2, P38 and JNK (Fig. 3A). However, the AP-1 proteins exhibited varying sensitivity to CSE (Fig. 3B). For example, p-c-Jun was more sensitive to $0.25 \%$ CSE, while Jun B was more sensitive to $0.1 \%$ CSE; whereas p-c-Fos was continuously activated by 
A

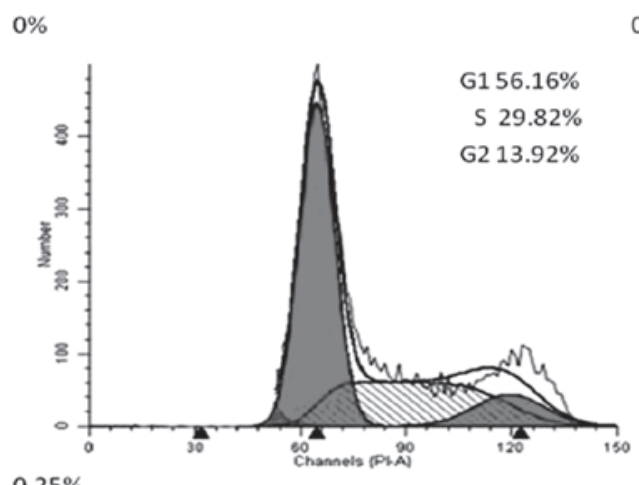

$0.1 \%$
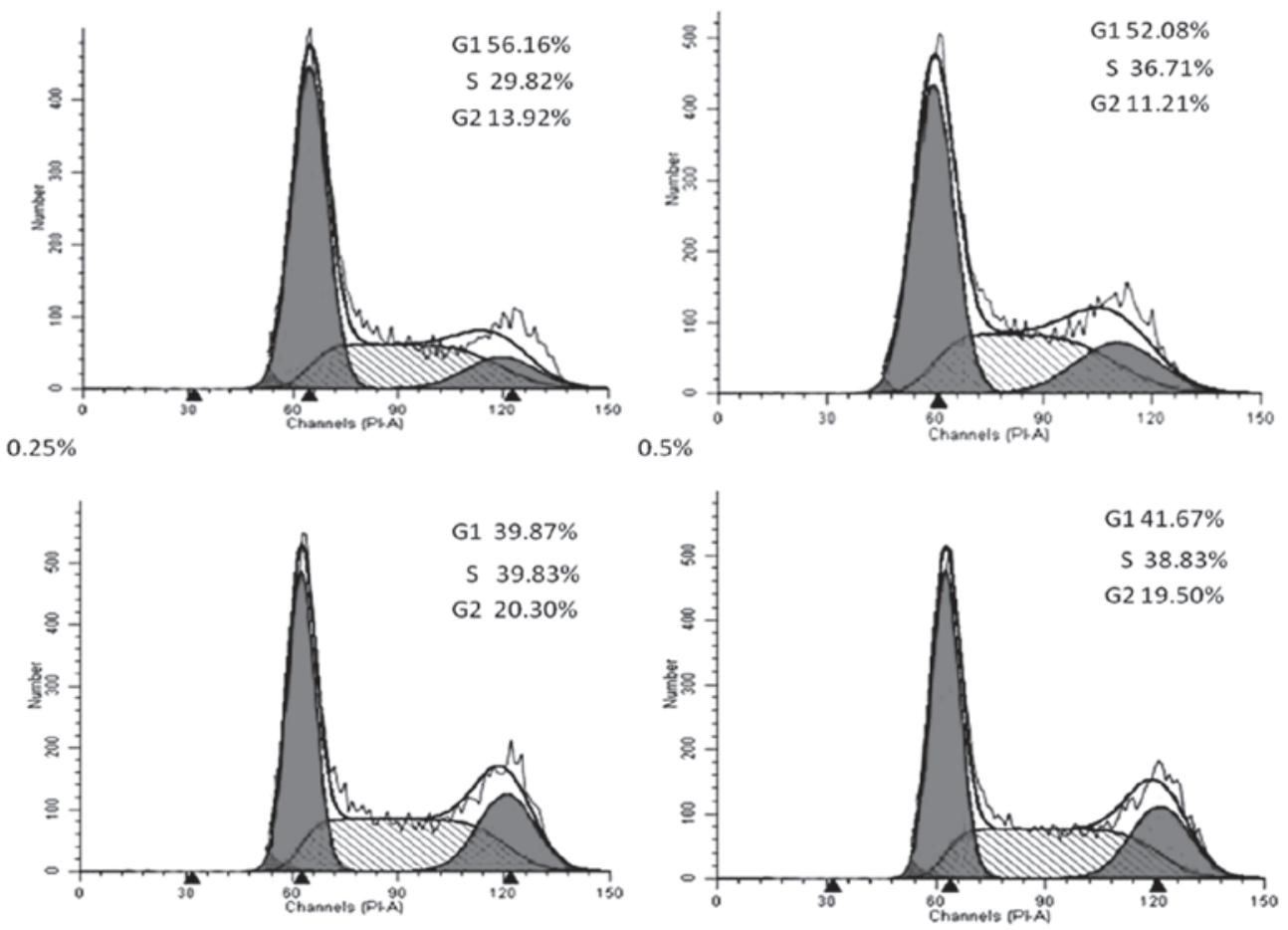

B

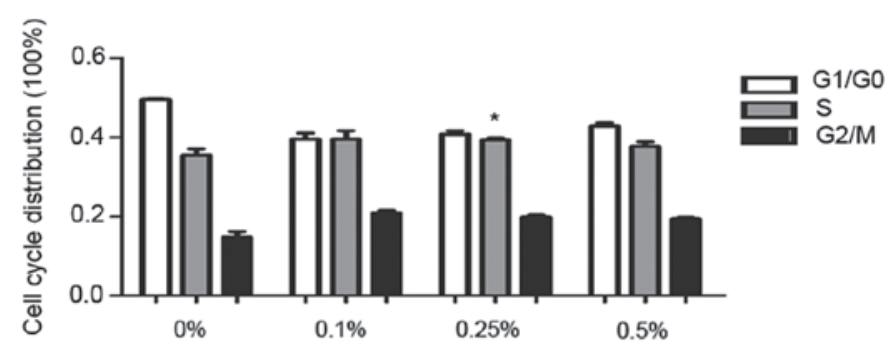

C

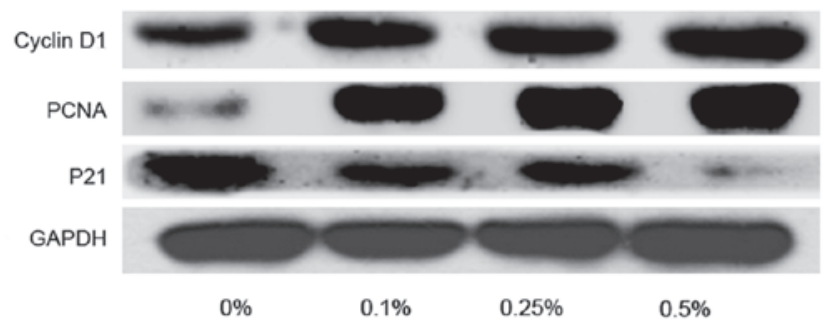

D
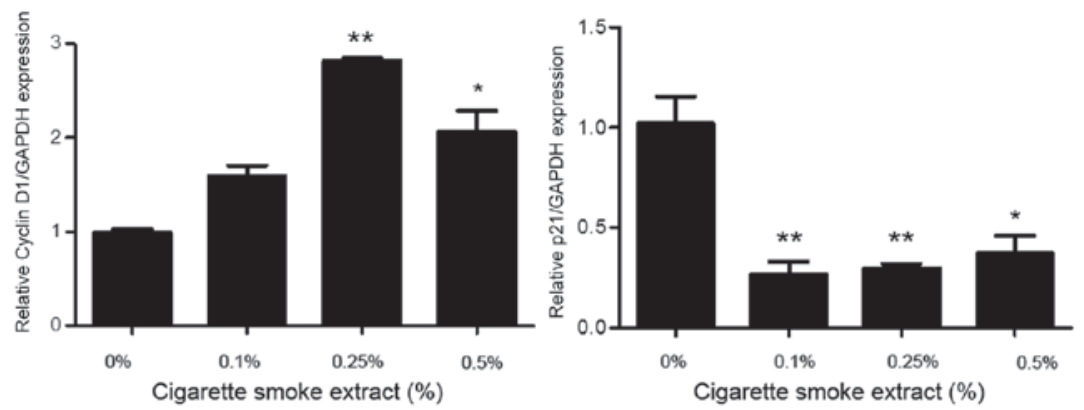

Figure 2. (A) CSE promoted the transformation of phase GO into G1 phase in SV-HUC-1 cells, particularly the 0.25\% CSE. (B) Cell cycle phase is shown in a bar graph form with the G0/G1, S and G2/M phases. Folowing tratment with $0.25 \%$ CSE, the number of SV-HUC-1 cells entering S phase (39.83\%) was significantly higher than that of the control $(29.82 \%)$. Data are representative of three independent experiments and are expressed as the mean \pm standard deviation. ${ }^{*} \mathrm{P}<0.05$ vs. control group. (C) Western blots and (D) quantification revealed the relative protein expression of cyclin D1, p21 and proliferating cell nuclear antigen in SV-HUC-1 cells following treatment with $0,0.10,0.25$ and $0.50 \%$ CSE for 7 days. ${ }^{*} \mathrm{P}<0.05$ and ${ }^{* *} \mathrm{P}<0.01$ vs. control group. CSE, cigarette smoke extract; mRNA, messenger RNA. 
A

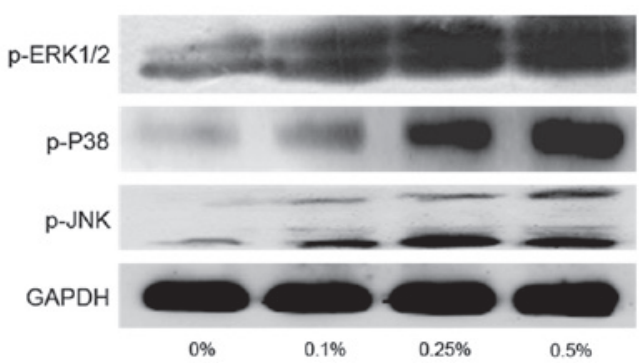

B

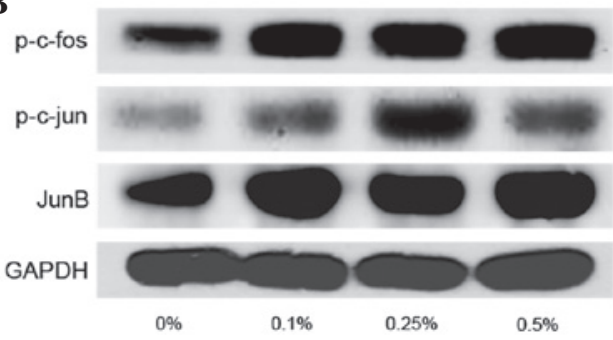

Figure 3. (A) Expression of proteins involved in the mitogen-activated protein kinase pathway, including p-ERK1/2, p-p38 and p-JNK, in SV-HUC-1 cells following treatment with $0.10,0.25$ and $0.50 \%$ CSE for 7 days. p-ERK1/2, p-P38 and p-JNK protein expression was increased following CSE treatment. (B) Expression of proteins involved in activator protein 1 pathway, including p-c-Jun, p-c-Fos, Jun B and Jun D in SV-HUC-1 cells following treatment with CSE for 7 days. The expression of p-c-Jun, p-c-Fos and Jun B were markedly increased following CSE treatment. p-, phosphorylated; ERK, extracellular signal regulated protein kinase; JNK, Jun N-terminal kinase; GAPDH, glyceraldehyde 3-phosphate dehydrogenase.

A

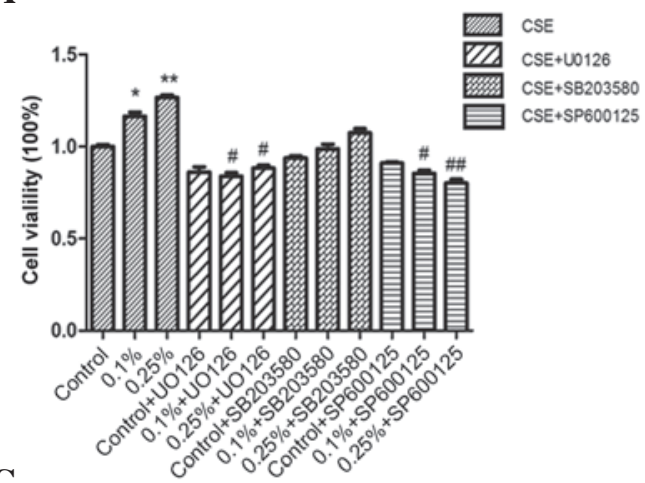

C

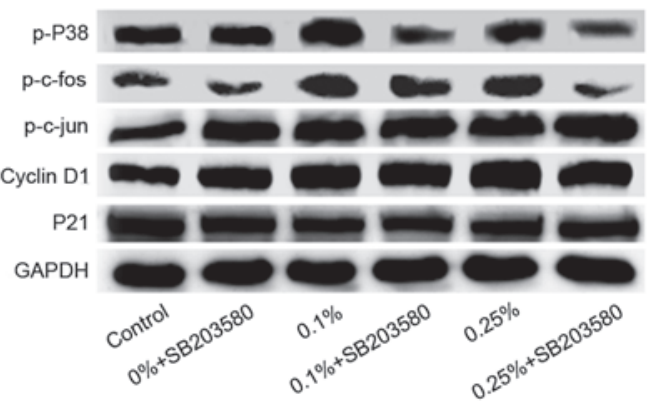

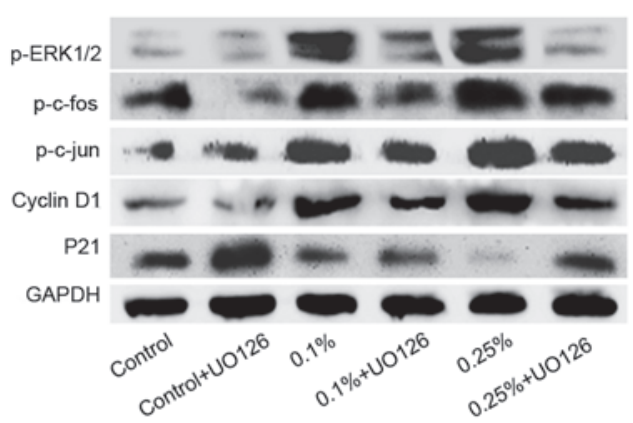

D

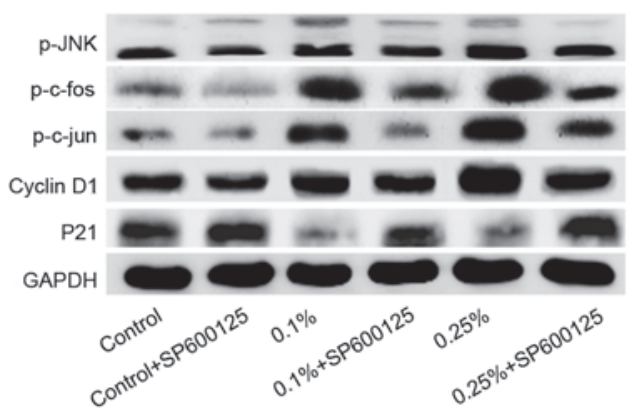

Figure 4. (A) Fold change in cell viability of SV-HUC-1 cells treated with $0,0.10$ and $0.25 \%$ CSE for 7 days in combination with $5 \mu \mathrm{M}$ U0126, $5 \mu \mathrm{M}$ SB203580 or $2 \mu \mathrm{M} \mathrm{SP} 600125$. Data are representative of three independent experiments and are expressed as the mean \pm standard deviation. ${ }^{*} \mathrm{P}<0.05$ vs. control group (0\% CSE + dimethyl sulfoxide); ${ }^{\#} \mathrm{P}<0.05$ represents CSE \pm inhibitor group vs. respective CSE group. (B) Protein expression of AP-1 pathway and cell cycle markers in SV-HUC-1 cells following combined treatment with CSE and $5 \mu \mathrm{M}$ U0126 for 7 days. The upregulated expression of p-c-Fos, p-c-Jun, cyclin D1 following CSE treatment was decreased by U0126, while U0126 inhibited the CSE-induced downregulation of p21. (C) Protein expression of AP-1 pathway and cell cycle markers in SV-HUC-1 cells following combined treatment with CSE and $5 \mu \mathrm{M}$ SB203580 for 7 days. The expression of p-c-Jun was decreased by SB203580. The expression of p-c-Fos and cell cycle regulators, cyclin D1 and p21, was not decreased following SB203580 treatment. (D) Protein expression of AP-1 pathway and cell cycle markers in SV-HUC-1 cells following combined treatment with CSE and $2 \mu \mathrm{M}$ SP600125 for 7 days. p-c-Fos, p-c-Jun, cyclin D1 and p21 were decreased following treatment with SP600125. CSE, cigarette smoke extract; p-, phosphorylated; ERK, extracellular signal regulated protein kinase; JNK, Jun N-terminal kinase; GAPDH, glyceraldehyde 3-phosphate dehydrogenase; AP-1, activator protein-1.

CSE, which indicates that at higher concentrations of CSE, the effects of Jun D attenuation are more evident.

MAPK pathway inhibitors reverse CSE-induced proliferation in SV-HUC-1 cells. To investigate the role of the MAPK pathway in CSE-induced cell proliferation of SV-HUC-1 cells, cells were incubated with DMSO (control group), 0.10 or $0.25 \%$ CSE in combination with U0126 $(5 \mu \mathrm{M})$, SB203580 $(5 \mu \mathrm{M})$ or SP600125 $(2 \mu \mathrm{M})$ for 7 days. An MTT assay was conducted to analyze cell viability and the inhibitory effects induced, following treatment with MAPK inhibitors (Fig. 4A). The results revealed that U0126 and SP600125 markedly reversed CSE-induced proliferation in SV-HUC-1 cells $(\mathrm{P}<0.001 ; 0.25 \% \mathrm{CSE}$ vs. $0.25 \% \mathrm{CSE}+\mathrm{U} 0126 / \mathrm{SP} 600125)$. SB203580 marginally attenuated the effect of CSE; however, the effect was not reversed, which indicates that p38 is not involved in the proliferative effects induced by CSE.

Western blot analysis revealed similar results. p-ERK1/2 was significantly downregulated and the expression of p-c-Jun and p-c-Fos was attenuated by U0126 treatment after 
CSE treatment (Fig. 4B). Furthermore, U0126 reversed the CSE-induced upregulation of cyclin D1 and downregulation of p21 expression. However, SB203580 exhibited no effect on cyclin D1 or p21 expression following CSE treatment (Fig. 4C). Additionally, SP600125 treatment markedly inhibited the effects of CSE, and the expression of downstream p-c-Fos and p-c-Jun were strongly inhibited. Furthermore, the expression of cyclin D1 and p21 were decreased following SP600125 treatment (Fig. 4D). Notably, following treatment with SP600125, the observed protein levels of cyclin D1 and p21 were similar to the levels exhibited in the control group, which indicates that SP600125 may be present a potential chemopreventative agent. U0126, SB203580 and SP600125 treatment exhibited little effect on the control group and mainly diminished the effect induced by CSE.

\section{Discussion}

The present study demonstrated that exposure to CSE activated the MAPK/AP-1 pathway and induced cell proliferation in SV-HUC-1 cells. Furthermore, inhibitors of the ERK1/2 and JNK pathways reversed the proliferation induced by exposure to CSE. However, the inhibition of p38 did not reverse the proliferation induced by CSE.

$\mathrm{BC}$ is one of the most common tumors of the urinary tract. It is a disease that peaks in older patients, and $~ 90 \%$ of cases are pathologically diagnosed as urothelial carcinoma (15). Cigarette smoke is considered to be the major risk factor for BC (3). Thus, elucidating the processes involved in the transformation of normal urothelial cells to malignant cells induced by exposure to cigarette smoke may aid the development of early targeted interventions for BC. To date, $>60$ carcinogens, which are present in mainstream smoke, sidestream smoke and the particulate phase of CSE, including polycyclic aromatic hydrocarbons, benzo[a]pyrene, nitrosamines and aromatic amines, have been identified (16). As the risk of $\mathrm{BC}$ is significantly associated with the intensity and duration of exposure to cigarette smoke, CSE was selected as a stimulus in the present study, as it has been widely used in previous BC studies $(17,18)$.

Previous studies have demonstrated that the MAPK/AP-1 pathway is involved in the effects induced after treatment with CSE $(6,19)$. The Ras-MAPK signaling pathway is a well-established target for anticancer therapies, due to its central role in regulating the growth and survival of cells (19). BC is a disease caused by alterations in several cellular processes, characterized by constitutive activation of the Ras-MAPK pathway (20). In the present study, CSE was found to activate the ERK1/2, P38 and JNK pathways. The activation of the ERK signaling pathway, also known as the p42/p44 MAP kinase pathway, is a major determinant in the control of cell growth, differentiation and survival (21). Furthermore, ERK1/2 inhibitors have been administered as anti-cancer drugs (22). The present study results revealed that ERK1/2 inhibition reversed the proliferative effects induced by CSE, which may aid with the development of novel therapies for bladder cancer. Notably, a previous study demonstrated that JNK and p38 are involved in arsenic-induced activating transcription factor 2 activation in SV-HUC-1 cells (23). However, few studies have investigated
CSE-induced AP-1 activation in SV-HUC-1 cells, as demonstrated in the present study. The results of the present study revealed that ERK1/2 and JNK regulated the expression of AP-1 proteins involved in the proliferative effects induced by CSE (Fig. 4B and D).

The AP-1 transcription factor is a hetero or homodimeric complex that comprises members of the proto-oncogene Jun (c-Jun, Jun B and Jun D) and Fos (c-Fos, Fos B, Fra-1 and Fra-2) protein families. c-Fos and c-Jun are a pair of transcription factors that, in combination with other associated proteins, form the AP-1 transcription factor complex (24). AP-1 exhibits a crucial role in the carcinogenesis of tumors via the promotion of cell proliferation, invasion and metastasis. c-Jun expression has been found to positively correlate with an increasing tumor stage in BC (25).

Bladder carcinogenesis has been reported to be initiated by the clonal expansion of genetically altered cells that include normal mucosa and premalignant lesions (26). In the present study, CSE treatment resulted in the upregulation of cyclin D1 and PCNA expression and the downregulation of p21 expression in SV-HUC-1 cells via the AP-1 pathway. Cyclin D1 and PCNA are AP-1 target genes that are involved in cell proliferation. Cyclin D1 gene regulatory sequences contain two AP-1 binding sites. Several AP-1 proteins, including c-Jun and c-Fos, bind these sites to subsequently activate cyclin D1 expression (27). Cyclin D1 is a gene that controls the cell cycle and promotes cell cycle progression through G1-phase by forming active holoenzymes with cyclin-dependent kinase (CDK)4 and CDK6, which leads to phosphorylation of retinoblastoma protein (28). Cyclin D1 overexpression has been demonstrated to be an independent adverse risk factor in metastasizing BC (29). Similarly, the PCNA gene contains AP-1 binding sites in the promoter region and thus its expression is regulated by AP-1 activity (30). Consistent with the results of the present study, a number of previous studies have demonstrated that exposure to cigarette smoke and nicotine induces the expression of PCNA $(31,32)$. Additionally, p21 is a well-characterized PCNA partner that has been identified in a protein complex that contains PCNA, cyclin D1 and CDK. The p21 protein exhibits two various inhibitory effects on the entry of a cell into S phase: Inhibition of the kinase activity of CDK and inhibition of DNA replication via interactions with PCNA (33). According to the results of the present study, ERK1/2 and JNK inhibition attenuated the proliferative effects triggered by CSE.

In addition, the present study indicated that the inhibition of p38 did not reverse the proliferative effect on SV-HUC-1 cells induced by CSE. As shown in Fig. 4C, SB203580 did not suppress the CSE-induced expression of p-c-Fos, which may indicate why the proliferative effects induced by CSE were not reversed following SB203580 treatment. However, numerous signaling pathways are associated with exposure to cigarette smoke, including the nuclear factor- $\kappa \mathrm{B}$ and phosphoinositide 3-kinase/protein kinase B pathways (31), therefore further study is required.

The results of the present study confirmed that exposure to CSE induces proliferation in normal human urothelial cells. The ERK1/2 and JNK pathways are important in the regulation of CSE-induced proliferation of SV-HUC-1 cells via the AP-1 pathway. These findings revealed the effects of 
CSE on the proliferation of normal human urothelial cells and also highlighted the importance of the MAPK/AP-1 pathway in the development of CSE-induced pathogenesis, which may provide novel evidence with regard to the molecular mechanisms of $\mathrm{BC}$ development.

\section{Acknowledgements}

The present study was supported by the National Natural Science Foundation of China (Beijing, China; grant nos. 1373005, 81072330 and 81202194) and the Priority Academic Program Development of Jiangsu Higher Education Institutions (Nanjing, China), the Anhui Public Welfare Research Linkage Plan (Hefei, China; grant no. 1501ld04045) and the Anhui Medical University Scientific Research Funds (Hefei, China; grant no. H0514).

\section{References}

1. Siegel R, Naishadham D and Jemal A: Cancer statistics, 2012. CA Cancer J Clin 62: 10-29, 2012.

2. Boffetta P: Tobacco smoking and risk of bladder cancer. Scand J Urol Nephrol Suppl: 45-54, 2008.

3. Zeegers MP, Kellen E, Buntinx F and van den Brandt PA: The association between smoking, beverage consumption, diet and bladder cancer: A systematic literature review. World J Urol 21: 392-401, 2004

4. Colli Neto JA, Zen Júnior JH, Del Negro A, Andreollo NA, Araujo MR and Tincani AJ: Tobacco experimental model to induce urinary bladder neoplasms. Rev Col Bras Cir 41: 56-60, 2014.

5. Hecht SS: Tobacco carcinogens, their biomarkers and tobacco-induced cancer. Nature Rev Cancer 3: 733-744, 2003.

6. Zhong CY, Zhou YM, Douglas GC, Witschi $\mathrm{H}$ and Pinkerton KE: MAPK/AP-1 signal pathway in tobacco smoke-induced cell proliferation and squamous metaplasia in the lungs of rats Carcinogenesis 26: 2187-2195, 2005.

7. Johnson GL and Lapadat R: Mitogen-activated protein kinase pathways mediated by ERK, JNK, and p38 protein kinases. Science 298: 1911-1912, 2002.

8. Seger R and Krebs EG: The MAPK signaling cascade. FASEB J 9: 726-735, 1995.

9. Montironi R, Cheng L, Scarpelli M, Mazzucchelli R and Lopez-Beltran A: How much do you know about benign, preneoplastic, non-invasive and invasive neoplastic lesions of the urinary bladder classified according to the 2004 WHO scheme? Diagn Pathol 6: 31, 2011.

10. Chu M, Guo J and Chen CY: Long-term exposure to nicotine, via ras pathway, induces cyclin D1 to stimulate G1 cell cycle transition. J Biol Chem 280: 6369-6379, 2005.

11. KosakaM,Kang MR, Yang GandLiLC: Targeted p21 (WAF1/CIP1) activation by RNAa inhibits hepatocellular carcinoma cells. Nucleic Acid Ther 22: 335-343, 2012.

12. Tian D, Zhu M, Chen WS, Li JS, Wu RL and Wang X: Role of glycogen synthase kinase 3 in squamous differentiation induced by cigarette smoke in porcine tracheobronchial epithelial cells. Food Chem Toxicol 44: 1590-1566, 2006.

13. Geng H, Zhao L, Liang Z, Zhang Z, Xie D, Bi L, Wang Y, Zhang T, Cheng L, Yu D and Zhong C: ERK5 positively regulates cigarette smoke-induced urocystic epithelial-mesenchymal transition in SV40 immortalized human urothelial cells. Oncol Rep 34: $1581-1588,2015$.
14. Livak KJ and Schmittgen TD: Analysis of relative gene expression data using real-time quantitative PCR and the 2(-Delta Delta C(T)) Method. Methods 25: 402-408, 2001.

15. Parkin DM, Bray F, Ferlay J and Pisani P: Global cancer statistics, 2002. CA Cancer J Clin 55: 74-108, 2005.

16. Hecht SS: Progress and challenges in selected areas of tobacco carcinogenesis. Chem Res Toxicol 21: 160-171, 2008.

17. Chen LM, Nergard JC, Ni L, Rosser CJ and Chai KX: Long-term exposure to cigarette smoke extract induces hypomethylation at the RUNX3 and IGF2-H19 loci in immortalized human urothelial cells. PLoS One 8: e65513, 2013.

18. Yang W, Cui S, Ma J, Lu Q, Kong C, Liu T and Sun Z: Cigarette smoking extract causes hypermethylation and inactivation of WWOX gene in T-24 human bladder cancer cells. Neoplasma 59: 216-223, 2012.

19. Sebolt-Leopold JS and Herrera R: Targeting the mitogen-activated protein kinase cascade to treat cancer. Nat Rev Cancer 4: 937-947, 2004.

20. Mitra AP and Cote RJ: Molecular pathogenesis and diagnostics of bladder cancer. Annu Rev Pathol 4: 251-285, 2009.

21. Kohno M and Pouyssegur J: Pharmacological inhibitors of the ERK signaling pathway: Application as anticancer drugs. Prog Cell Cycle Res 5: 219-224, 2003.

22. Zhao L, Geng H, Liang ZF, Zhang ZQ, Zhang T, Yu DX and Zhong CY: Benzidine induces epithelial-mesenchymal transition in human uroepithelial cells through ERK1/2 pathway. Biochem Biophys Res Commun 459: 643-649, 2015.

23. Liu S, Wang F, Yan L, Zhang L, Song Y, Xi S, Jia J and Sun G: Oxidative stress and MAPK involved into ATF2 expression in immortalized human urothelial cells treated by arsenic. Arch Toxicol 87: 981-989, 2013.

24. Hess J, Angel P and Schorpp-Kistner M: AP-1 subunits: Quarrel and harmony among siblings. J Cell Sci 117: 5965-5973, 2004

25. Tiniakos DG, Mellon K, Anderson JJ, Robinson MC, Neal DE and Horne $\mathrm{CH}$ : c-jun oncogene expression in transitional cell carcinoma of the urinary bladder. Br J Urol 74: 757-761, 1994.

26. Crawford JM: The origins of bladder cancer. Lab Invest 88: 686-693, 2008.

27. Brown JR, Nigh E, Lee RJ, Ye H, Thompson MA, Saudou F, Pestell RG and Greenberg ME: Fos family members induce cell cycle entry by activating cyclinD1. Mol Cell Biol 18: 5609-5619, 1998.

28. Weinberg RA: The retinoblastoma protein and cell cycle control. Cell 81: 323-330, 1995.

29. Seiler R, Thalmann GN, Rotzer D, Perren A and Fleischmann A: CCND1/CyclinD1 status in metastasizing bladder cancer: A prognosticator and predictor of chemotherapeutic response. Mod Pathol 27: 87-95, 2014.

30. Gillardon F, Moll I and Uhlmann E: Inhibition of c-Fos expression in the UV-irradiated epidermis by topical application of antisense oligodeoxynucleotides suppresses activation of proliferating cell nuclear antigen. Carcinogenesis 16: 1853-1856, 1995

31. Deng QF, Sun X, Liang ZF, Zhang ZQ, Yu DX and Zhong CY: Cigarette smoke extract induces the proliferation of normal human urothelial cells through the NF- $\kappa \mathrm{B}$ pathway. Oncol Rep 35: 2665-2672, 2016.

32. Xia S, Kang J, Jiang Y, Huang D, Wang S and Pang B: Simvastatin promotes alveolar epithelial cell proliferation and attenuates cigarette smoke-induced emphysema in rats. Mol Med Rep 12: 5903-5910, 2015.

33. Waga S, Hannon GJ, Beach D and Stillman B: The p21 inhibitor of cyclin-dependent kinases controls DNA replication by interaction with PCNA. Nature 369: 574-578, 1994. 